\title{
Temperature variability and extremes both affect economic growth
}

\author{
Christopher W. Callahan ${ }^{1,2 *}$ \& Justin S. Mankin²,3,4 \\ ${ }^{1}$ Graduate Program in Ecology, Evolution, Environment and Society, Dartmouth College, Hanover, NH, USA \\ ${ }^{2}$ Department of Geography, Dartmouth College, Hanover, NH, USA \\ ${ }^{3}$ Department of Earth Sciences, Dartmouth College, Hanover, NH, USA \\ ${ }^{4}$ Ocean and Climate Physics, Lamont-Doherty Earth Observatory of Columbia University, Palisades, NY, USA \\ *Corresponding author: Christopher Callahan, Christopher.W.Callahan.GR@dartmouth.edu
}

Arising from Kotz et al. (2021), "Day-to-day temperature variability reduces economic growth," Nature Climate Change, https://doi.org/10.1038/s41558-020-00985-5

This manuscript is a non-peer-reviewed preprint that has been submitted for publication in Nature Climate Change. Subsequent versions of this manuscript may have different content. If accepted, the final version of this manuscript will be available via the "Peer-reviewed Publication DOI" link on the right-hand-side of this webpage. Please feel free to contact the corresponding author with questions or comments at Christopher.W.Callahan.GR@dartmouth.edu or @cwcallahan45. 
Changes in temperature averages, variability, and extremes may all independently affect economic growth under climate change. Kotz et al. ${ }^{1}$ show that temperature variability reduces growth, but find no significant effect of temperature extremes. Recreating their results, here we show that temperature extremes do indeed affect growth independently from the effects of variability. Our results emphasize the need to consider multiple related moments of the temperature distribution when analyzing climate damages and to use metrics of temperature extremes that are relative to the local climate.

Understanding the effect of climate on economic growth is necessary to develop optimal mitigation and adaptation policy ${ }^{2}$. Recent analyses have shown that temperature increases can substantially reduce growth ${ }^{3,4}$, thereby increasing optimal emissions reductions ${ }^{5}$. These studies focused on annual mean temperature, but parts of the economy such as agriculture, mortality, and labor productivity exhibit non-linear and threshold-like responses to temperature that may not be fully captured by its annual mean ${ }^{6-8}$. Furthermore, extreme temperatures are projected to increase faster than annual means ${ }^{9}$. As such, estimating growth effects solely based on annual means might mask the effect of temperature extremes, which is how people and economies may most directly experience climate change.

To address this problem, Kotz et al. ${ }^{1}$ analyze sub-national temperature variability and persuasively show that it reduces growth. They further argue that variability damages growth independently from the increased occurrence of temperature extremes during years of high variability. Temperature distributions are often non-Gaussian and can have long tails ${ }^{10}$, so the relationship between average temperatures, variability, and extremes is often complex ${ }^{11}$ and warrants further study. Kotz et al. ${ }^{1}$ incorporate extremes by examining the linear effect of degree days relative to strict thresholds of 25 and $30{ }^{\circ} \mathrm{C}$. Using those indices, the authors find no significant effect of extreme temperatures on growth. Here we extend their analysis by using a more flexible definition of extreme temperatures and allowing for heterogeneity in its effects. We find a spatially heterogeneous yet robust effect of extremes on economic growth, one that accounts for a portion of the effect of temperature variability.

Our empirical model mirrors the specification of Kotz et al. ${ }^{1}$, including temperature variability, its interaction with the magnitude of the regional annual cycle, and several other temperature variables (Supplementary Information). We extend their analysis by including extreme degree days (EDDs), a metric that measures accumulated degree days above percentile-based thresholds. Contrasted with the Kotz et al. ${ }^{1}$ approach of using temperature thresholds of 25 and $30{ }^{\circ} \mathrm{C}$, our percentile-based definitions are relative, meaning they are defined differently for each location and day of the year. We focus on the 98 th percentile, meaning that only $2 \%$ of days for that location and day of the year are warmer. This definition incorporates adaptations undertaken by societies with respect to their local climates and the timing of extreme temperatures ${ }^{12}$. A given temperature may be far more damaging to an agricultural region during grain filling than other times of the year, for example. We interact EDDs with the annual cycle, mirroring the Kotz et al. ${ }^{1}$ interaction of variability, with one difference: for EDDs we include both linear and quadratic interactions with the annual cycle (rather than only linear interactions). We make this choice because the quadratic interaction is highly significant for EDDs, but not for variability. All marginal effects presented here are standardized by the average within-region standard deviation of the relevant variable. Further details are provided in the Supplementary Information.

We find a positive effect of extreme temperatures on growth in regions with large annual temperature cycles, such as the mid- to high-latitudes, along with an increasingly negative effect of extremes in low-annual-cycle regions, such as the tropics (Fig. 1a, Table 1, Table S1). In re- 
gions where the amplitude of the annual cycle is large, like $35{ }^{\circ} \mathrm{C}$ or more (such as Russia), an additional standard deviation (s.d.) of extreme temperatures increases growth by 1.03 percentage points (p.p.). But in regions where the annual cycle is low, like $5{ }^{\circ} \mathrm{C}$ (such as Brazil), extremes decrease growth by 1.34 p.p. These effects are similar when including a control for region-specific growth trends (Table S1). Using a 95th percentile thresholds yields smaller but still significant effects (Table S1). Consistent with Kotz et al. ${ }^{1}$, the effect of temperature variability is negative at all values of the annual cycle (Fig. 1a), with greater effects in regions with smaller annual cycles.

We use the relationship between the annual cycle and the marginal effects of extreme temperature to infer these effects for each region, even where growth data is unavailable (Supplementary Information; hatched regions in Fig. $1 \mathrm{~b}$ are out-of-sample estimates). The effects of extremes have a clear latitudinal structure: tropical countries experience substantial declines in growth from temperature extremes due to their limited annual cycle, whereas mid-latitude countries gain (Fig. 1b). The mechanism for this structure may be similar to that proposed in Kotz et al. ${ }^{1}$, whereby countries that experience broader annual cycles (i.e., mid- and high-latitude regions) are better adapted to wide temperature distributions and therefore more severe extremes. At the same time, narrower annual cycles are linked to higher temperatures and lower incomes (i.e., the tropics). It is also possible that higher baseline temperatures make extremes more damaging by crossing physiological thresholds ${ }^{13}$, or that lower incomes make tropical countries less resilient to extremes ${ }^{12}$. A combination of all of these mechanisms is likely at work.

Kotz et al. ${ }^{1}$ show that the effect of variability is exactly unchanged when including linear effects of degree days above 25 and $30^{\circ} \mathrm{C}$, suggesting its independence from extreme temperatures (their
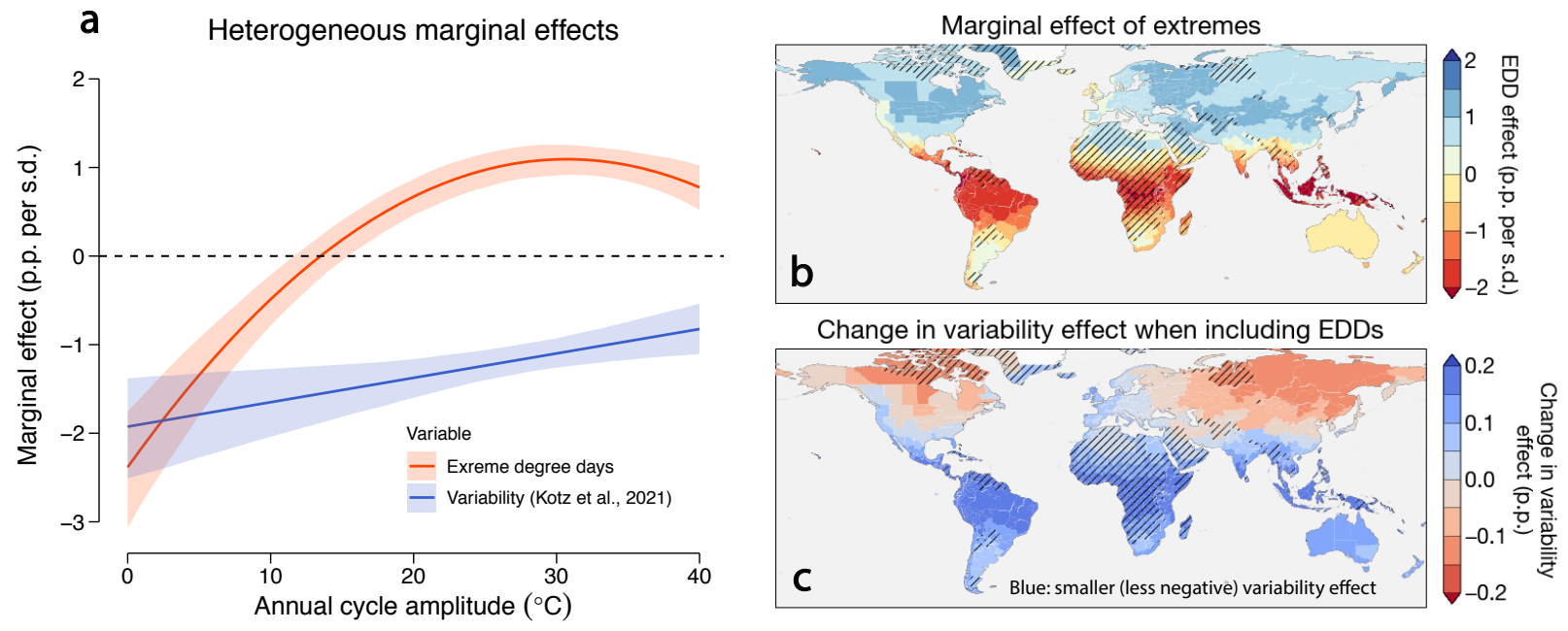

Figure 1: Effects of temperature extremes and variability on economic growth. a) Marginal effects of extreme degree days (EDD) and temperature variability on economic growth across a range of average annual cycle amplitudes (Supplementary Information). Solid line shows the main effect and shading shows the $95 \%$ confidence intervals, calculated with a bootstrap resample that is blocked by region (Supplementary Information). b) Marginal effects of extreme temperatures for each subnational region, calculated based on that region's average annual cycle. Hatching denotes regions where GDP data is not available and marginal effects are inferred using climate data. c) Change in the marginal effect of temperature variability between the baseline model of Kotz et al. ${ }^{1}$ and our model including EDDs (Table S1, column 1 vs. column 3). Blue regions are regions where the effect becomes less negative. 
Table S1). However, we find that including EDDs causes the baseline effect of variability to be reduced from -0.115 p.p. per ${ }^{\circ} \mathrm{C}$ to -0.103 p.p. per ${ }^{\circ} \mathrm{C}$ in our preferred specification (Table 1 ). In regions with a small annual cycle, temperature extremes may account for up to $10 \%$ of the negative effect of variability (Fig. 1c). On the other hand, including extremes also causes the interaction between variability and the annual cycle to decline, so in regions with large annual cycles, including extremes actually makes variability more damaging (Fig. 1c). It is possible that variability alone has some independent negative effect in these cold regions with large annual cycles, but the effects of warm extreme temperatures (which could benefit crops or cold-related mortality) may slightly mask this effect.

We emphasize that, consistent with Kotz et al. ${ }^{1}$, the negative effect of variability is large and significant in all our specifications (Fig. 1a and Table S1). Therefore, these results complement and enhance the basic findings of Kotz et al. ${ }^{1}$, reaffirming that the effects of extremes and variability are generally independent. In contrast to Kotz et al. ${ }^{1}$, however, we find that the effect of variability decreases when including a more flexible metric of extreme temperatures. This change demonstrates that some of the explanatory power of temperature variability may have been misestimated and may instead be attributable to greater extreme temperatures in years with greater variability.

Here we extend and clarify the analysis of Kotz et al. ${ }^{1}$, showing that temperature extremes and temperature variability both affect economic growth. Climate change is expected to increase heat extremes ${ }^{14}$ and may alter temperature variability in complex ways ${ }^{15}$, so understanding the effect of various moments of the temperature distribution on economic growth is essential. Combined with evidence that higher average temperatures could also reduce growth ${ }^{3,4}$ and that temperature extremes are increasing faster than other moments of the distribution ${ }^{9}$, these findings make the case for increased climate adaptation and mitigation ambition even more urgent.

\begin{tabular}{lcc}
\hline & Kotz et al. model & Our preferred model \\
\hline Variability & $-0.1150^{* * *}$ & $-0.103^{* * *}$ \\
& $(0.0148)$ & $(0.0153)$ \\
Variability $\times$ annual cycle & $0.0019^{* * *}$ & $0.0015^{* *}$ \\
& $(0.0005)$ & $(0.0005)$ \\
EDD & & $-0.005^{* * *}$ \\
& & $(0.0007)$ \\
EDD $\times$ annual cycle & & $0.0005^{* * *}$ \\
& & $(0.0001)$ \\
EDD $\times$ annual $\operatorname{cycle}^{2}$ & $-7.8 \mathrm{e}-6^{* * *}$ \\
& & $(0.0000)$ \\
\hline$* * * p<0.001 ;{ }^{* *} p<0.01 ;{ }^{*} p<0.05$ & &
\end{tabular}

Table 1: Selected regression coefficients in the original Kotz et al. model and our preferred model. Each coefficient denotes the percentage-point change in growth per 1-unit change in independent variable. The full models and the rest of the coefficients are shown in Table S1. Our preferred specification is column 3 of Table S1. 


\title{
Supplementary Material for "Temperature variability and extremes both affect economic growth"
}

\author{
Christopher W. Callahan \& Justin S. Mankin
}

\section{Methods}

Data

We use ERA5 reanalysis data ${ }^{16}$ from 1979-2018 to calculate extreme degree days (EDDs). EDDs are calculated by first finding the temperature value that corresponds to a desired percentile threshold (e.g., $98^{\text {th }}$ percentile) independently for each grid cell and day of the year. We use the entire 1979-2018 period as the reference period for calculating these thresholds. EDDs in a given year are then calculated as the accumulated sum of degrees above the desired threshold, similar to a "growing degree days" calculation (e.g., ref. ${ }^{17}$ ). We exclude days that do not fall in a series of three consecutive days above the desired threshold, to focus on the most damaging multi-day periods of extreme heat ${ }^{18}$.

We then calculate population-weighted area means over the regions used in Kotz et al. ${ }^{1}$, using the shapefile polygons they helpfully provided with their replication data and population data from the Gridded Population of the World ${ }^{19}$. EDDs at the region-year level are then merged with the replication data provided by Kotz et al. ${ }^{1}$.

There are occasional outliers that may disproportionately affect the estimation, so when using EDDs referenced to the $98^{\text {th }}$ percentile, we exclude observations with more than 50 EDDs. We do the same for the $95^{\text {th }}$ percentile and 75 EDDs. In both cases these thresholds correspond to limiting out less than $1 \%$ of the sample, and the results are not substantially different if the sample is not limited (Table $\mathrm{S} 1$ ).

\section{Empirical approach}

The preferred model used in Kotz et al. ${ }^{1}$ (column 7 of their Table 1 ) is as follows:

$$
g_{r t}=\beta_{1} V_{r t}+\beta_{2} V_{r t} * A_{r}+\beta_{3} \delta T_{r t}+\beta_{4} \delta T_{r t} * \bar{T}_{r t}+\beta_{5} \delta T_{r(t-1)}+\beta_{6} \delta T_{r(t-1)} * \bar{T}_{r t}+P_{r t}+\mu_{r}+\nu_{t}+\epsilon_{r t}
$$

where $V$ refers to the annual average within-month standard deviation of daily temperature, $A$ refers to the amplitude of a region's annual cycle (calculated as the maximum monthly temperature minus the minimum monthly temperature, averaged over all years), $\delta T$ refers to the change in temperature relative to the previous year, $\bar{T}$ refers to the long-term mean of temperature, and $P$ refers to precipitation. Here we focus on our analysis on variability and the annual cycle and do not further analyze the average temperature terms. Our analysis adds extreme temperatures to their baseline model (equation 1):

$$
g_{r t}=\alpha_{1} E D D_{r t}+\alpha_{2} E D D_{r t} * A_{r}+\alpha_{3} E D D_{r t} * A_{r}^{2}+\ldots+\epsilon_{r t}
$$

EDD refers to extreme degree days, as detailed in Data. In our main model we used EDDs referenced to the $98^{\text {th }}$ percentile, but also test the $95^{\text {th }}$ percentile (Table S1). The EDD term is interacted with the regional annual cycle and its square to allow the effect of extremes to vary by region. The quadratic term is highly significant when interacted with EDDs, but not significant when interacted with variability, so our preferred model has linear and quadratic interactions for EDDs but only a linear interaction for variability. 
We test the inclusion of a region-specific linear trend in growth (Table S1, column 5), which is a strategy that has been used by other papers to control for smooth, time-variant cofounders, and we find that our results are not substantially altered. However, we share the concern for overfitting expressed by Kotz et al. ${ }^{1}$, so we avoid these linear trends in our preferred specification.

\section{Plotting marginal effects}

The marginal effect of extreme temperatures for each region is calculated as:

$$
\frac{\partial g_{r t}}{\partial E D D_{r t}}=\alpha_{1}+\alpha_{2} * A_{r}+\alpha_{3} * A_{r}^{2}
$$

Similarly, the marginal effect of variability is calculated as:

$$
\frac{\partial g_{r t}}{\partial V_{r t}}=\beta_{1}+\beta_{2} * A_{r}
$$

Marginal effects are scaled by the average interannual standard deviation in each variable across regions to standardize the effect (4.74 for EDDs, 0.187 for variability). 95\% confidence intervals on the marginal effect are generated with 1000 iterations of bootstrap resampling, where we resample by region to preserve autocorrelation in regions across time (which mirrors clustering standard errors by region).

Not all regions have GDP data, so the sample used for the estimation does not include most regions in Africa, some regions in South America, and other missing areas, though climate data is available for these regions (see Fig. 2 of Kotz et al. ${ }^{1}$ ). We choose to map marginal effects for all regions, so we calculate each region's effect based on its annual cycle and show all regions in Fig. 1. The hatching denotes regions where this out-of-sample extrapolation has been performed. The primary regions of extrapolation are in the tropics (e.g., Africa), which are areas of with low annual cycles. The estimation sample contains regions with annual cycles as low as $2{ }^{\circ} \mathrm{C}$, so most of the regions for which we calculate marginal effects are within the range of the data covered by the estimation sample.

Fig. 1a plots marginal effects across values of the annual cycle from 0 to $40{ }^{\circ} \mathrm{C}$, and we observe a plateau of the effect above approximately $25{ }^{\circ} \mathrm{C}$. The quadratic interaction results in a strong decline in the marginal effect of extremes above $40{ }^{\circ} \mathrm{C}$, which we do not believe is realistic. Rather, we believe the best way to interpret the curve in Fig. 1a is a strong non-linear effect at low values of annual cycle and a plateau above $25-30{ }^{\circ} \mathrm{C}$. Hence, for all regions with annual cycles greater than $40{ }^{\circ} \mathrm{C}$, we set their marginal effects to be equal to the effect at $40^{\circ} \mathrm{C}$. This is a relatively small portion of the sample, primarily concentrated in northern Russia, so we do not believe it should substantially alter our results. 


\begin{tabular}{|c|c|c|c|c|c|}
\hline & \multicolumn{5}{|c|}{ Dependent variable: Growth } \\
\hline & (1) & $(2)$ & $(3)$ & $(4)$ & $(5)$ \\
\hline Variability & $\begin{array}{c}-0.115^{* * *} \\
(0.0148)\end{array}$ & $\begin{array}{c}-0.1041^{* * *} \\
(0.0153)\end{array}$ & $\begin{array}{c}-0.103^{* * *} \\
(0.0153)\end{array}$ & $\begin{array}{c}-0.1047^{* * *} \\
(0.0155)\end{array}$ & $\begin{array}{c}-0.1096^{* * *} \\
(0.0160)\end{array}$ \\
\hline Variability $\times$ seasonality & $\begin{array}{c}0.0019^{* * *} \\
(0.0005)\end{array}$ & $\begin{array}{l}0.0015^{* *} \\
(0.0005)\end{array}$ & $\begin{array}{l}0.0015^{* *} \\
(0.0005)\end{array}$ & $\begin{array}{l}0.0015^{* *} \\
(0.0005)\end{array}$ & $\begin{array}{l}0.0015^{* *} \\
(0.0005)\end{array}$ \\
\hline EDD & & $\begin{array}{c}-0.0045^{* * *} \\
(0.0006)\end{array}$ & $\begin{array}{c}-0.005^{* * *} \\
(0.0007)\end{array}$ & $\begin{array}{c}-0.0019^{* * *} \\
(0.0003)\end{array}$ & $\begin{array}{c}-0.0049^{* * *} \\
(0.0007)\end{array}$ \\
\hline EDD $\times$ seasonality & & $\begin{array}{c}0.0004^{* * *} \\
(0.0001)\end{array}$ & $\begin{array}{c}0.0005^{* * *} \\
(0.0001)\end{array}$ & $\begin{array}{c}0.0002^{* * *} \\
(0.0000)\end{array}$ & $\begin{array}{c}0.0005^{* * *} \\
(0.0001)\end{array}$ \\
\hline EDD $\times$ seasonality $^{2}$ & & $\begin{array}{c}-7.4 \mathrm{e}-6^{* * *} \\
(0.0000)\end{array}$ & $\begin{array}{c}-7.8 \mathrm{e}-6^{* * *} \\
(0.0000)\end{array}$ & $\begin{array}{c}-2.5 \mathrm{e}-6^{* * *} \\
(0.0000)\end{array}$ & $\begin{array}{c}-7.3 \mathrm{e}-6^{* * *} \\
(0.0000)\end{array}$ \\
\hline$\delta \mathrm{T}$ & $\begin{array}{c}0.0021 \\
(0.0021)\end{array}$ & $\begin{array}{l}-0.0038 \\
(0.0022)\end{array}$ & $\begin{array}{c}-0.0044^{*} \\
(0.0022)\end{array}$ & $\begin{array}{c}-0.0053^{*} \\
(0.0024)\end{array}$ & $\begin{array}{l}-0.0039 \\
(0.0023)\end{array}$ \\
\hline$\delta \mathrm{T}_{t-1}$ & $\begin{array}{c}-0.0004 \\
(0.0021)\end{array}$ & $\begin{array}{l}-0.0037 \\
(0.0022)\end{array}$ & $\begin{array}{l}-0.0041 \\
(0.0022)\end{array}$ & $\begin{array}{l}-0.0042 \\
(0.0022)\end{array}$ & $\begin{array}{l}-0.0028 \\
(0.0023)\end{array}$ \\
\hline$\delta \mathrm{T} \times \overline{\mathrm{T}}$ & $\begin{array}{c}-0.0010^{* * *} \\
(0.0002)\end{array}$ & $\begin{array}{c}-0.0008^{* * *} \\
(0.0002)\end{array}$ & $\begin{array}{c}-0.0007^{* * *} \\
(0.0002)\end{array}$ & $\begin{array}{c}-0.0006^{* *} \\
(0.0002)\end{array}$ & $\begin{array}{c}-0.0007^{* * *} \\
(0.0002)\end{array}$ \\
\hline$\delta \mathrm{T}_{t-1} \times \overline{\mathrm{T}}$ & $\begin{array}{c}-0.0007^{* * *} \\
(0.0002)\end{array}$ & $\begin{array}{c}-0.0006^{* *} \\
(0.0002)\end{array}$ & $\begin{array}{c}-0.0006^{* *} \\
(0.0002)\end{array}$ & $\begin{array}{c}-0.0005^{*} \\
(0.0002)\end{array}$ & $\begin{array}{c}-0.0007^{* *} \\
(0.0002)\end{array}$ \\
\hline Precipitation & $\begin{array}{c}0.0003 \\
(0.0001)\end{array}$ & $\begin{array}{l}0.0003^{*} \\
(0.0001)\end{array}$ & $\begin{array}{l}0.0003^{*} \\
(0.0001)\end{array}$ & $\begin{array}{c}0.0003 \\
(0.0001)\end{array}$ & $\begin{array}{c}0.0006^{* * *} \\
(0.0001)\end{array}$ \\
\hline Region-specific growth trend & No & No & No & No & Yes \\
\hline EDD percentile threshold & & $98^{\text {th }}$ & $98^{\text {th }}$ & $95^{\text {th }}$ & $98^{\text {th }}$ \\
\hline Sample restriction & No & No & $\mathrm{EDD}<50$ & $\mathrm{EDD}<75$ & $\mathrm{EDD}<50$ \\
\hline Observations & 28,874 & 27,338 & 27,283 & 27,113 & 27,283 \\
\hline Adjusted $\mathrm{R}^{2}$ & 0.226 & 0.231 & 0.2301 & 0.226 & 0.240 \\
\hline
\end{tabular}

Supplementary Table 1: Effect of temperature variability and extremes on growth. Column 1 replicates column 7 of Table 1 in Kotz et al. (2021). Region-clustered standard errors are presented in parentheses. Region and year fixed effects are included in all specifications. Sample restriction is discussed in the Methods section of the Supplementary Information. Table created using the texreg package in $\mathrm{R}^{20}$. 


\section{References}

[1] Kotz, M., Wenz, L., Stechemesser, A., Kalkuhl, M. \& Levermann, A. Day-to-day temperature variability reduces economic growth. Nature Climate Change 1-7 (2021).

[2] Diaz, D. \& Moore, F. Quantifying the economic risks of climate change. Nature Climate Change 7, 774-782 (2017).

[3] Dell, M., Jones, B. F. \& Olken, B. A. Temperature shocks and economic growth: Evidence from the last half century. American Economic Journal: Macroeconomics 4, 66-95 (2012).

[4] Burke, M., Hsiang, S. \& Miguel, E. Global non-linear effect of temperature on economic production. Nature 527, 235-239 (2015).

[5] Moore, F. C. \& Diaz, D. B. Temperature impacts on economic growth warrant stringent mitigation policy. Nature Climate Change 5, 127 (2015).

[6] Barreca, A., Clay, K., Deschenes, O., Greenstone, M. \& Shapiro, J. S. Adapting to climate change: The remarkable decline in the US temperature-mortality relationship over the twentieth century. Journal of Political Economy 124, 105-159 (2016).

[7] Graff Zivin, J. \& Neidell, M. Temperature and the allocation of time: Implications for climate change. Journal of Labor Economics 32, 1-26 (2014).

[8] Schlenker, W. \& Roberts, M. J. Nonlinear temperature effects indicate severe damages to US crop yields under climate change. Proceedings of the National Academy of Sciences 106, 15594-15598 (2009).

[9] Coffel, E. D., Horton, R. M., Winter, J. M. \& Mankin, J. S. Nonlinear increases in extreme temperatures paradoxically dampen increases in extreme humid-heat. Environmental Research Letters 14, 084003 (2019).

[10] Ruff, T. W. \& Neelin, J. D. Long tails in regional surface temperature probability distributions with implications for extremes under global warming. Geophysical Research Letters 39 (2012).

[11] Huybers, P., McKinnon, K. A., Rhines, A. \& Tingley, M. Us daily temperatures: The meaning of extremes in the context of nonnormality. Journal of Climate 27, 7368-7384 (2014).

[12] Carleton, T. A. et al. Valuing the global mortality consequences of climate change accounting for adaptation costs and benefits. NBER working paper (2020).

[13] Coffel, E. D., Horton, R. M. \& de Sherbinin, A. Temperature and humidity based projections of a rapid rise in global heat stress exposure during the 21st century. Environmental Research Letters 13, 014001 (2017).

[14] Meehl, G. A. \& Tebaldi, C. More intense, more frequent, and longer lasting heat waves in the 21st century. Science 305, 994-997 (2004).

[15] Fischer, E. M., Rajczak, J. \& Schär, C. Changes in European summer temperature variability revisited. Geophysical Research Letters 39 (2012).

[16] C3S. ERA5: Fifth generation of ECMWF atmospheric reanalysis of the global climate. Copernicus Climate Change Service Climate Data Store (2017). 
[17] Butler, E. E., Mueller, N. D. \& Huybers, P. Peculiarly pleasant weather for US maize. Proceedings of the National Academy of Sciences 115, 11935-11940 (2018).

[18] Perkins, S. E. \& Alexander, L. V. On the measurement of heat waves. Journal of Climate 26, 4500-4517 (2013).

[19] Center for International Earth Science Information Network, C. U., CIESIN. Gridded population of the world, version 4 (gpwv4): Population count. Online (2016).

[20] Leifeld, P. texreg: Conversion of statistical model output in $\mathrm{R}$ to $\mathrm{LAT}_{\mathrm{EX}}$ and HTML tables. Journal of Statistical Software 55, 1-24 (2013). URL http://dx.doi.org/10.18637/jss.v055.i08. 